\title{
COMMUNICATION
}

Cite this: DOI: $10.1039 / x 0 x x 00000 x$

\section{Amorphous metal-organic frameworks for drug delivery}

Received 00th January 2012, Accepted 00th January 2012

DOI: $10.1039 / \times 0 \times x 00000 x$

www.rsc.org/

\author{
Claudia Orellana-Tavra, ${ }^{\mathrm{a}}$ Emma F. Baxter, ${ }^{\mathrm{b}}$ Tian Tian, ${ }^{\mathrm{a}}$ Thomas D. Bennett, ${ }^{\mathrm{b}}$ \\ Nigel K. H. Slater, ${ }^{\mathrm{a}}$ Anthony K. Cheetham, ${ }^{\mathrm{b}}$ and David Fairen-Jimenez ${ }^{\mathrm{a}, *}$
}

We report the encapsulation of the hydrophilic model molecule calcein in the Zr-based MOF UiO-66, followed by amorphization of the framework by ball-milling. We show controlled release of calcein over more than 30 days, compared with the 2 day release period from crystalline $\mathrm{UiO}$ 66.

Drugs in the form of chemical and bioactive compounds are widely used as therapeutic agents to improve health and to extend the lifespan of the human population. Many of these compounds are successful candidates for the treatment of severe illnesses such as cancer. However, they can also have significant disadvantages, including poor solubility and nonselective biodistribution, which often results in the damage of healthy tissues $^{1}$ and cardiotoxicity effects, ${ }^{2,3}$ which strongly limits their therapeutic potential. By making use of a drug delivery system (DDS), most of these issues can be overcome through the increment of drug solubility, protection from degradation, controlled drug release, provision of targeted delivery, and a decrease in toxic side effects. Finding an effective DDS for therapeutic agents has been an ongoing challenge in bioengineering. In this context, metal-organic frameworks (MOFs) have emerged as potential candidates owing to their distinctive characteristics, such as high pore volumes, large surface areas, multiple topologies and tuneable pore size and surface chemistry. ${ }^{4,5}$ MOFs are synthesized in a self-assembly process from metal ions or clusters, acting as coordination centres that are interconnected by organic ligands.

In recent years, MOFs have proven to be able to encapsulate and delivery drugs efficiently by adsorbing them in their pore structures. Amongst others, Horcajada et al. have loaded different anticancer and antiviral agents into MOFs; ${ }^{6}$ Morris et al. encapsulated and delivered the vasodilator agent nitric oxide gas (NO); ${ }^{7}$ Lin et al. reported the use of MOFs for the codelivery of the anticancer cisplatin molecule, and siRNA to enhance therapeutic effect. ${ }^{8}$ Some of the principal advantages of MOFs compared with other organic (e.g. liposomes and micelles) and inorganic (e.g. zeolites and mesoporous silicas) DDSs are their high loading capacities and the possibility of chemically functionalizing the materials to enhance drug affinity and to target cells. Very recently, we performed a computational screening study showing how MOFs can encapsulate up to $2 \mathrm{~g}$ of a drug per gram of porous solid, a much higher capacity than what can be found in mesoporous silicas and organic carriers - typically up to $0.3 \mathrm{mg} / \mathrm{g} .{ }^{9,10}$

The relatively poor chemical stability of the MOF family tempers many of their advantages for industrial applications. Such chemical instability can be considered advantageous in drug delivery processes, since, unlike e.g. mesoporous silicas, MOF DDS can be easily biodegraded in the body after the drug has been released. Limitations in their use do still remain however, such as very fast kinetic deliveries of under 3 days from a variety of MOF-drug systems. .,11 $^{2}$

An increasing awareness of amorphous MOFs ${ }^{12}\left(a_{\mathrm{m}} \mathrm{MOFs}\right.$, i.e. highly disordered framework structures retaining the basic metal-ligand connectivity of crystalline MOFs, though lacking any long range order) in recent years has led to the use of ballmilling to trap guest molecules by irreversibly collapsing porous networks around occluded species such as molecular $\mathrm{I}_{2}{ }^{13,14}$ Building on this concept, we hypothesized that loading of a therapeutic agent, followed by subsequent structural collapse by ball-milling, could be used to achieve prolonged controlled release in solution. In this scenario, the delivery process will be at least partially controlled by material degradation (i.e. dissolution) in addition to drug diffusion through the porous network.

In order to probe this hypothesis, we selected in this study calcein as a model drug due to its structural similarities to doxorubicinin, a well-known anti-cancer drug. We also choose calcein due to its hydrophilic character, which means it cannot 
cross cell membranes, and therefore requires a DDS to enter into cells. In addition, being a fluorescent molecule, it can be easily traced by confocal microscopy and, due to its selfquenching characteristics, high local concentrations of calcein (e.g. when it is adsorbed in a MOF before being delivered) cannot be detected until it is released from the material. ${ }^{15} \mathrm{We}$ used the UiO-66 MOF, $\left[\mathrm{Zr}_{6} \mathrm{O}_{4}(\mathrm{OH})_{4}(\mathrm{BDC})_{6}\right] \quad(\mathrm{BDC}=1,4-$ benzenedicarboxylate) as a DD vehicle. UiO-66 has a cubic structure based on $\mathrm{Zr}$ oxo-clusters and BDC ligands, ${ }^{16}$ and possesses high thermal and chemical stability combined with a large porosity $\left(\mathrm{S}_{\mathrm{BET}}=1200 \mathrm{~m}^{2} \mathrm{~g}^{-1}, \mathrm{~V}_{\mathrm{p}}=0.5 \mathrm{~cm}^{3} \mathrm{~g}^{-1}\right)$ formed by two main cavities ( $c a .11$ and $\left.8 \AA \varnothing_{\text {cavity }}\right) .{ }^{17}$ Additionally, $\mathrm{Zr}$ has low toxicity (zirconyl acetate lethal dose $\mathrm{LD}_{50} \sim 4.1 \mathrm{mg} / \mathrm{ml}$ in rats; furthermore, the human body contains $\sim 300 \mathrm{mg}$ of $\mathrm{Zr}$ and the amount daily ingested is $\sim 3.5 \mathrm{mg} /$ day). ${ }^{18}$

We first loaded calcein into UiO-66 by suspending the activated MOF into a calcein low concentration solution producing the cal@UiO-66 sample, which retained the structure of the original UiO-66 after drug loading (Fig. 1). Subsequent ball-milling on the empty UiO-66 framework produced an Xray amorphous product which we term cal@ $a_{\mathrm{m}} \mathrm{UiO}-66$. Figure S1 (electronic supporting information, ESI) shows the scanning electron microscopy (SEM) pictures of UiO-66 and cal@ $a_{\mathrm{m}} \mathrm{UiO}-66$, with particle sizes of $261 \pm 12$ and $272 \pm 157$ $\mathrm{nm}$, respectively. The morphology of the particles after amorphization is clearly less homogeneous, which we ascribe to particle compaction during the intense mechanical ballmilling process. Figure S2 shows the thermogravimetric analysis (TGA) of UiO-66, with a first weight lost below $100^{\circ} \mathrm{C}$, which corresponds to the desorption of solvent molecules, followed by the solid degradation at ca. $475^{\circ} \mathrm{C}$. Additionally, cal@UiO-66 shows another step at $c a .400^{\circ} \mathrm{C}$, which corresponds to the calcein desorption from the material. The amount of calcein loaded, measured by TGA, was $4.9 \pm 0.2$ wt.\%. In comparison, degradation of free calcein occurs at $165^{\circ} \mathrm{C}$ approximately. These results show the successful adsorption of calcein in UiO-66. We decided not to increase the calcein loading in order to avoid self-quenching and aid confocal microscopy imaging (vide infra).

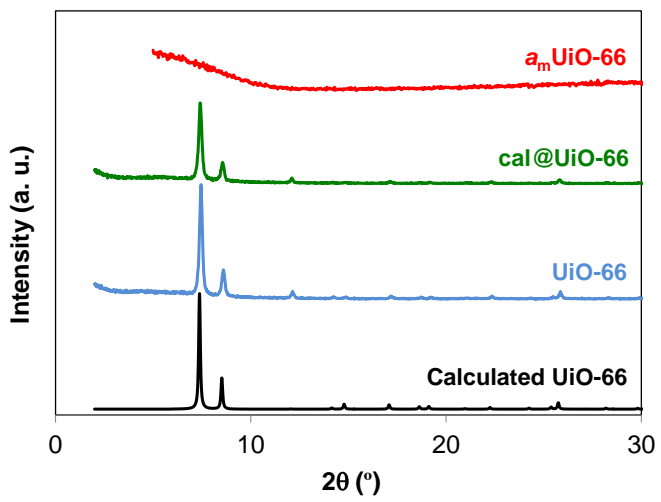

Figure 1. Powder X-ray diffraction (PXRD) patterns of synthesized UiO-66, cal@UiO-66 and cal@ $a_{\mathrm{m}}$ UiO-66 compared with the calculated one for UiO-66.
Figure 2 and S3 show the drug delivery profiles obtained for cal@UiO-66 and cal@ $a_{\mathrm{m}} \mathrm{UiO}-66 . \mathrm{UiO}-66$ releases all the calcein within 2 days; similar values have been found already for caffeine on UiO-66. ${ }^{17}$ Interestingly, $a_{\mathrm{m}} \mathrm{UiO}-66$ shows a significantly slower, sustained release, prolonging the delivery time up to 30 days, i.e. an order of magnitude higher than its crystalline counterpart. Indeed, the calcein releases by the crystalline and amorphous frameworks during the first hour are ca. 36 and $3.5 \%$, respectively. After 5 hours, these amounts are increased to 68 and $16 \%$ for UiO-66 and $a_{\mathrm{m}} \mathrm{UiO}-66$, respectively, and after 10 days, $a_{\mathrm{m}} \mathrm{UiO}-66$ still retains $33 \%$ of calcein. Figure S4 shows the comparison between UiO-66 and $a_{\mathrm{m}} \mathrm{UiO}-66$ samples after 10 days release, where the presence of calcein in $a_{\mathrm{m}} \mathrm{UiO}-66$ is still evident.

The delivery time for cal@UiO-66 is aligned with the release times presented in literature for other drug-MOF systems. ${ }^{6}$ In contrast, we were able to extend the release time in the $a_{\mathrm{m}} \mathrm{UiO}-$ 66 sample by using the ball-milling process. In addition to the TGA results, the extended release time suggests that calcein was effectively adsorbed inside the material and not only in the external surface. Fitting of release patterns revealed a simple hyperbolic curve for UiO-66, whereas $a_{\mathrm{m}} \mathrm{UiO}-66$ showed a two term expression (full details can be found in the SI). During the first stage in $a_{\mathrm{m}} \mathrm{UiO}-66$, release of calcein takes place presumably through desorption and diffusion along the amorphous pore texture of the material. The second release stage is a much slower process that might be associated with the partial dissolution of defects in the $a_{\mathrm{m}} \mathrm{UiO}-66$ porous texture to liberate the remnants of the encapsulated calcein. Further work to analyse the release of calcein and dissolution of $a_{\mathrm{m}} \mathrm{UiO}-66$ is in progress. These results confirm that it is possible to use the amorphization process to trap a drug inside the material and to achieve a controlled, slower release.

To the best of our knowledge, such extremely long release times have not been reported before for any MOF or DDS with similar characteristics. For example, by using a core-shell microsphere with a diameter of $c a .400 \mu \mathrm{m}$, it has been possible to extend the release time of a water soluble drug up to 5 days. ${ }^{19}$ The use of core-shell biodegradable microfibers has permitted to extended the delivery of the anticancer Paclitaxel (PTX) for approximately 5 days. $^{20}$ Also, hydrogel systems
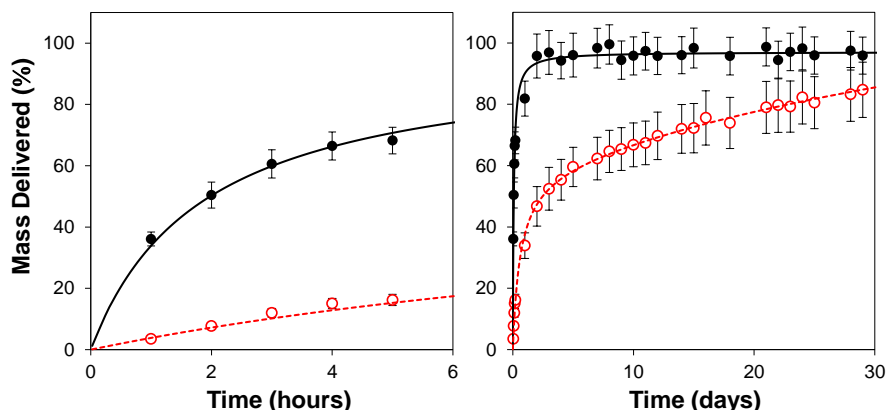

Figure 2. (left) Calcein release during the first 5 hours and (right) 30 days from UiO-66, black closed circles, and $a_{\mathrm{m}} \mathrm{UiO}-66$, red open circles. Black solid and red dotted lines represent the kinetics of delivery, fitted using non-linear regression on UiO-66 and $a_{\mathrm{m}} \mathrm{UiO}-66$, respectively. 
have been used for controlled delivery showing an important correlation between the network structure and the release rate. Indeed, hydrogel microspheres of 3-5 $\mu \mathrm{m}$ of diameter showed release time prolonged for 13 days. However, when using larger hydrogel cylinders, drug release was increased up to 200 days. ${ }^{21}$ All these systems are useful options only in the case of cell membrane permeable drugs and non-parenteral routes of administration. This is because, in contrast with our proposed $a_{\mathrm{m}} \mathrm{MOF}$ system, the size of the carriers is not small enough (i.e. $\sim 200 \mathrm{~nm}$ ) to enter into the cells or to circulate through the smallest capillaries.

To evaluate the biocompatibility, drug transport and the way the crystalline and amorphous UiO-66 interact with cells crossing their membranes, we moved to in vitro studies in HeLa cells, which is a widely studied culture. Figure S5 shows the cytotoxicity activity of UiO-66, with $\mathrm{IC}_{50}$ values of $1.503 \pm$ $0.154 \mathrm{mg} / \mathrm{ml}$ and $1.357 \pm 0.088 \mathrm{mg} / \mathrm{ml}$ for 24 and $48 \mathrm{~h}$, respectively. Similar values are obtained for $a_{\mathrm{m}} \mathrm{UiO}-66$. Zirconyl acetate lethal dose $\mathrm{LD}_{50}$ is reported to be $4.1 \mathrm{mg} / \mathrm{ml}$ in rats. ${ }^{18} \mathrm{We}$ consider this values promising for using UiO-66 in healthcare applications.

Figure $3 \mathrm{a}$ and $3 \mathrm{~b}$ show the confocal microscopy images of HeLa cells incubated for 24 and $48 \mathrm{~h}$, respectively, for: i) the fixed cell control; non-fixed cells incubated with ii) free calcein; iii) cal@UiO-66, and iv) cal@ $a_{\mathrm{m}} \mathrm{UiO}-66$. We used Hoechst 33342 (H33342) and propidium iodide (PI) for staining the nucleus of the cells and as a viability control, respectively. PI is unable to stain cells with intact membrane and has been widely used to assess the viability of cells. ${ }^{22}$ For fixed cells which have lost their cell membrane integrity, PI successfully stained them. However, for non-fixed cells, no PI signal was detected, indicating that the membranes of the cells containing the loaded UiO-66 were intact and consequently the cells were alive. This is particularly important in order to assess the crossing of cell the membrane by free drugs and DDS. Cells incubated with free calcein were not stained at $24 \mathrm{~h}$ of incubation and were only stained weakly in the form of bright vesicles at $48 \mathrm{~h}$ of incubation, suggesting that the dye is encapsulated in endosomes. Although calcein is believed not to penetrate the cell membrane, cellular uptake of impermeable dyes by endocytosis has been reported previously using Human Mesenchymal Stem Cells (MSCs) and the dye Lucifer yellow (LYCH). ${ }^{23}$ When the cells were incubated with cal@UiO-66, a strong signal was detected at $24 \mathrm{~h}$, and the signal was intensified at $48 \mathrm{~h}$ because of the rapid delivery of the drug. This confirms that the cal@UiO-66 complex was effectively incorporated into the cells. The confocal images show punctate staining that suggests MOF entrapment within intracellular vesicles. Cells incubated with cal@ $a_{\mathrm{m}} \mathrm{UiO}-66$ only showed a very weak signal after $24 \mathrm{~h}$ due to the self-quenching characteristic of calcein, and the signal was intensified at $48 \mathrm{~h}$. Both at 24 and $48 \mathrm{~h}$, the signal from cal@ $a_{\mathrm{m}} \mathrm{UiO}-66$ was less intense than that obtained from crystalline cal@UiO-66. This difference is due to the slower release of calcein from the amorphous material, $a_{\mathrm{m}} \mathrm{UiO}-66$, as observed previously in the
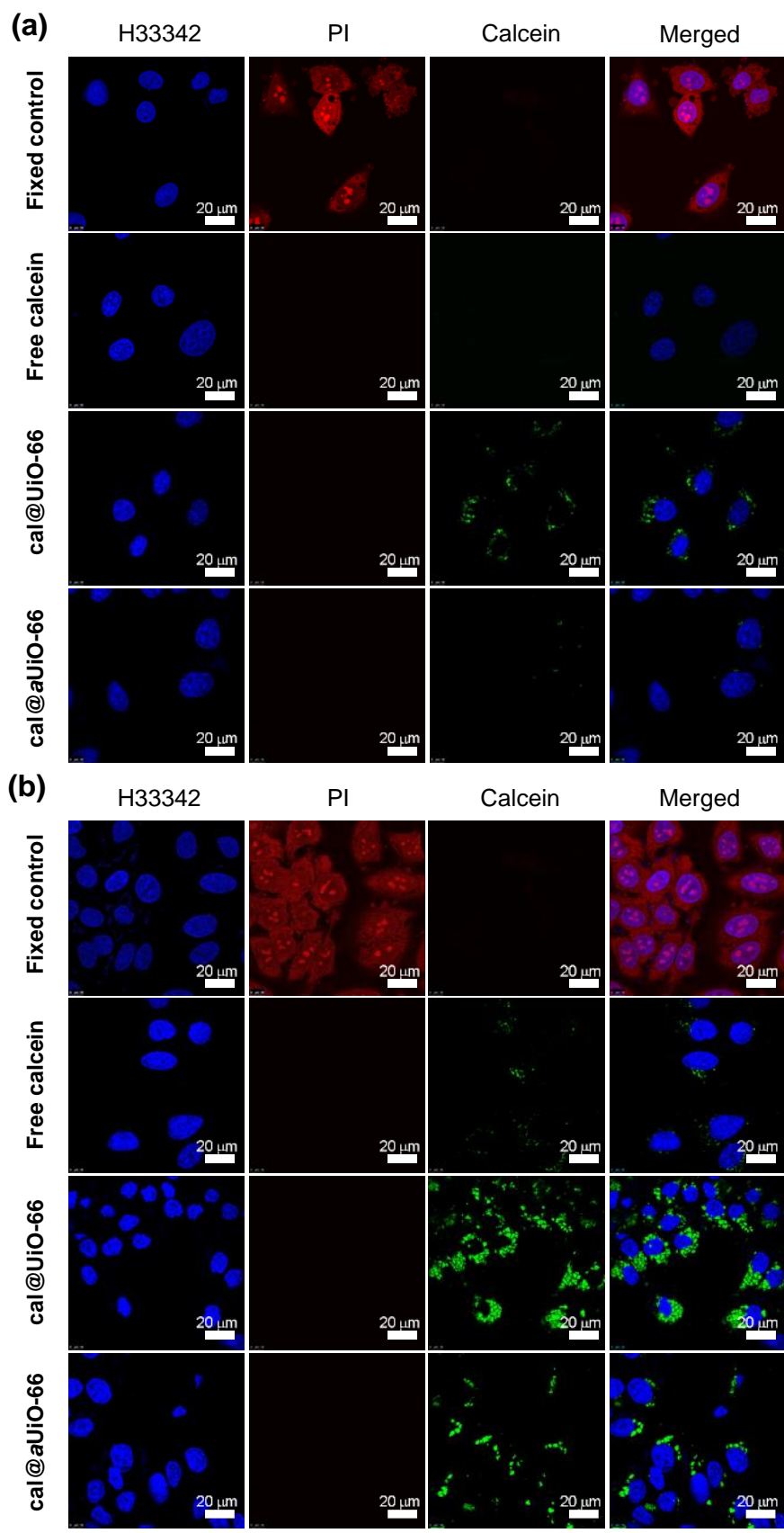

Figure 3. Confocal microscopy images of HeLa cells incubated for (a) 24 and (b) $48 \mathrm{~h}$. Cells were subsequently stained with Hoechst $33342(5 \mu \mathrm{g} / \mathrm{ml})$ and PI (5 $\mu \mathrm{g} / \mathrm{ml})$.

release assays. Additionally, for both forms of the material, the calcein signal detected is always stronger than the one obtained channel images are presented to have an integrated picture of the particle uptake by HeLa cells.

In order to quantify the amount of internalised complex cal@UiO-66 and cal@ $a_{\mathrm{m}} \mathrm{UiO}-66$, we performed flow cytometry measurements. Figure $4 \mathrm{a}$ and $\mathrm{b}$ show the two colour dot plot of HeLa cells incubated for $24 \mathrm{~h}$ for cal@UiO-66 and cal@ $a_{\mathrm{m}} \mathrm{UiO}-66$, respectively. We used CellTrace calcein AM red/orange as viability control because it is retained by cells with intact cell membrane. The y-axis represents the 
fluorescence intensity of CellTrace, whereas the $\mathrm{x}$-axis represents the fluorescence of calcein due to the uptake of cal@UiO-66 or cal@ $a_{\mathrm{m}} \mathrm{UiO}-66$. Figure 4c shows the average normalised calcein fluorescence of live cells that internalised either cal@UiO-66 or cal@ $a_{\mathrm{m}} \mathrm{UiO}-66$. On one hand, cytoplasmic calcein fluorescence from free calcein is $95 \%$ and 92\% lower thancal@UiO-66 and cal@ $a_{\mathrm{m}} \mathrm{UiO}-66$, respectively. This is due to the less efficient internalisation of free calcein because its hydrophilic character. On the other hand, crystalline cal@UiO-66 shows stronger cytoplasmic calcein fluorescence than that of cal@ $a_{\mathrm{m}} \mathrm{UiO}-66$ (41\% lower for cal@ $a_{\mathrm{m}} \mathrm{UiO}-66$ compared to cal@UiO-66). As explained before this phenomenon is a consequence of the self-quenching characteristics of calcein and the faster release of cal@UiO-66 compared to cal@a $a_{\mathrm{m}} \mathrm{UiO}-66$.
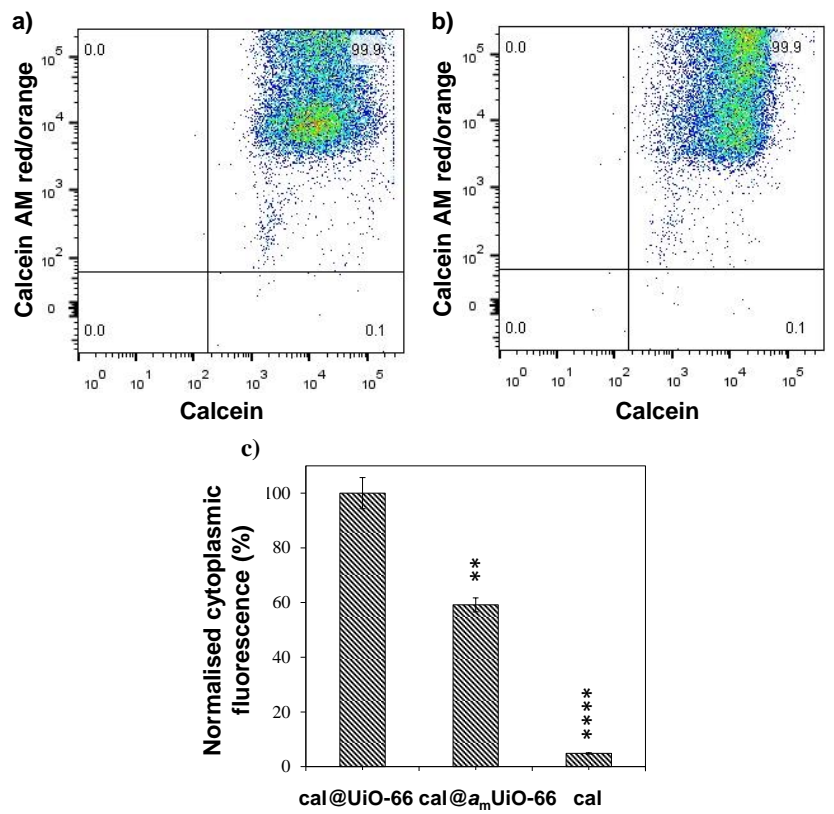

Figure 4. Flow cytometry two colour dot plot of HeLa cells incubated for $24 \mathrm{~h}$ with a) cal@UiO-66, and b) cal@ $a_{\mathrm{m}} \mathrm{UiO}-66$; c) shows the cytoplasmic calcein fluorescence of cells incubated with cal@UiO-66, cal@a $a_{\mathrm{m}}$ UiO-66 and free calcein. Error bars represent the standard error of three samples. Asterisks indicate: ** $\mathrm{P}$ $\leq 0.01$ and $* * * * \mathrm{P} \leq 0.0001$ in comparison with the cal@UiO-66 (Student's test).

\section{Conclusions}

We have successfully loaded and released the hydrophilic model drug calcein into the Zr-based MOF UiO-66. We have shown how the structural collapse of UiO-66 around calcein through a ball-milling amorphization process allows the entrapment of the drug inside the porosity. By doing this, we were able to significantly increase drug release times from 2 to up to 30 days in a drug delivery system (DDS) with a particle size of $272 \pm 157 \mathrm{~nm}$. Such extremely long times have not been reported before for any MOF or other DDS with similar characteristics. This striking increase in the length of the delivery period is in principle a generally applicable technique not only to other MOFs but also other crystalline materials. Finally, we are reporting crystalline and amorphous DDS not only able to extend the release time but to penetrate into the cells while maintaining the kinetic characteristic of the delivery. This feature is extremely useful especially for impermeable drugs and drugs with poor solubility. Future work with active therapeutic agents to study the biological effect of the system is currently ongoing.

\section{Notes and references}

${ }^{a}$ Department of Chemical Engineering \& Biotechnology, University of Cambridge, CB2 3RA Cambridge, United Kingdom.

${ }^{b}$ Department of Materials Science and Metallurgy, University of Cambridge, CB3 0FS Cambridge, United Kingdom

Electronic Supplementary Information (ESI) available: Synthesis, characterization data, amorphization process, calcein loading and delivery assays, and in vitro study information. See DOI: 10.1039/c000000x/ C.A.O. thanks Becas Chile and the Cambridge Trust for funding. T.D.B. thanks Trinity Hall (University of Cambridge) for funding. D.F.-J. thanks the Royal Society (UK) for funding through a University Research Fellowship. A.K.C is grateful to the European Research Council for an Advanced Investigator Award. We thank Mrs. Maggie Wallduck and Mr. Sergio Mercado for fruitful discussions.

1. L. Meng, X. Zhang, Q. Lu, Z. Fei, and P. J. Dyson, Biomaterials, 2012, 33, 1689.

2. R. Erttmann, N. Erb, A. Steinhoff, and G. Landbeck, Cancer Res. Clin. Oncol., 1988, 114, 509.

3. K. Shan, A. M. Lincoff, and J. B. Young, Am. Coll. Physicians, 1996, $125,47$.

4. H. Furukawa, K.E. Cordova, M. O'Keeffe, O.M. Yaghi, Science, 2013, 341, 974.

5. P. Horcajada, R. Gref, T. Baati, P. K. Allan, G. Maurin, P. Couvreur, G. Férey, R. E. Morris, and C. Serre, Chem. Rev., 2012, 112, 1232.

6. P. Horcajada, T. Chalati, C. Serre, B. Gillet, C. Sebrie, T. Baati, J. F. Eubank, D. Heurtaux, P. Clayette, C. Kreuz, J.-S. Chang, Y. K. Hwang, V. Marsaud, P.-N. Bories, L. Cynober, S. Gil, G. Férey, P. Couvreur, and R. Gref, Nat. Mater., 2010, 9, 172.

7. A. C. McKinlay, B. Xiao, D. S. Wragg, P. S. Wheatley, I. L. Megson, and R. E. Morris, J. Am. Chem. Soc., 2008, 130, 10440.

8. C. He, K. Lu, D. Liu, and W. Lin, J. Am. Chem. Soc., 2014, 136, 5181.

9. M. C. Bernini, D. Fairen-Jimenez, M. Pasinetti, A. J. Ramirez-Pastor, and R. Q. Snurr, J. Mater. Chem. B, 2014, 2, 766.

10. J. I. Jin-gou, Z. Jing-fen, H. A. O. Shi-lei, W. U. Dan-jun, L. I. U. Li, and X. U. Yi, 2012, 28, 166.

11. The only exception is the delivery of ibuprofen from MIL-53, with up to 21 days release. Nevertheless, caffeine is release from the same material in PBS within only 6 hours. See: D. Cunha, M. Ben Yahia, S. Hall, S. R. Miller, H. Chevreau, E. Elka, G. Maurin, P. Horcajada, C. Serre, Chem. Mater. 2013, 25, 2767.

12. T. D. Bennett and A. K. Cheetham, Acc. Chem. Res., 2014, 47, 1555.

13. T. D. Bennett, S. Cao, J. C. Tan, D. A. Keen, E. G. Bithell, P. J. Beldon, T. Friscic, and A. K. Cheetham, J. Am. Chem. Soc., 2011, 133, 14546.

14. T. D. Bennett, P. J. Saines, D. A. Keen, J.-C. Tan, and A. K. Cheetham, Chem.-Eur. J., 2013, 19, 7049.

15. M. Javadi, W. G. Pitt, C. M. Tracy, J. R. Barrow, B. M. Willardson, J. M. Hartley, and N. H. Tsosie, J. Control. Release, 2013, 167, 92.

16. J. H. Cavka, S. Jakobsen, U. Olsbye, N. Guillou, C. Lamberti, S. Bordiga, and K. P. Lillerud, J. Am. Chem. Soc., 2008, 130, 13850.

17. D. Cunha, C. Gaudin, I. Colinet, P. Horcajada, G. Maurin, and C. Serre, J. Mater. Chem. B, 2013, 1, 1101 .

18. H. Greim, in Zirconium and its compounds, The MAK Collection for Occupational Health and Safety, 1999, pp. 224-236.

19. M. P. A. Lim, W. L. Lee, E. Widjaja, and S. C. J. Loo, Biomater. Sci., 2013, 1, 486.

20. H.-H. Huang, C.-L. He, H.-S. Wang, and X.-M. Mo, J. Biomed. Mater. Res., 2009, 90, 1243.

21. A. Bertz, S. Wöhl-Bruhn, S. Miethe, B. Tiersch, J. Koetz, M. Hust, H. Bunjes, and H. Menzel, J. Biotechnol., 2013, 163, 243.

22. D. M. C. Sharp, A. Picken, T. J. Morris, C. J. Hewitt, K. Coopman, and N. K. H. Slater, Cryobiology, 2013, 67, 305.

23. A. Oliver, K. Jamil, J. Crowe, F. Tablin, and O. Al, Cell Preserv. Technol., 2004, 2, 35. 